\title{
Erratum to: The need of standardization and of large clinical studies in an emerging indication of [18 F]FDG PET: the autoimmune encephalitis
}

\author{
Silvia Morbelli ${ }^{1} \cdot$ Javier Arbizu $^{2} \cdot$ Jan Booij $^{3} \cdot$ Ming-Kai Chen $^{4} \cdot$ Gael Chetelat $^{5,6,7,8}$. $^{1}$ \\ Donna J. Cross $^{9}$ • Mehdi Djekidel ${ }^{10,11}$ - Alexander Drzezga ${ }^{12}$ • Ozgul Ekmekcioglu ${ }^{13}$. \\ Valentina Garibotto ${ }^{14}$ - Swen Hesse ${ }^{15}$ - Kazunari Ishii ${ }^{16}$ • Lida Jafari ${ }^{17}$. \\ Adriaan A. Lammertsma ${ }^{18} \cdot$ Ian Law $^{19}$ - Dana Mathews ${ }^{20} \cdot$ Satoshi Minoshima $^{9}$.

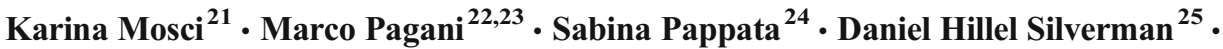 \\ Alberto Signore $^{26}$ • Elsmarieke Van De Giessen ${ }^{3}$ • Victor Villemagne Vi,28,29 $^{\text {. }}$ \\ Henryk Barthel ${ }^{15}$ on behalf of the European Association of Nuclear Medicine (EANM) \\ and of the Society of Nuclear Medicine and Molecular Imaging (SNMMI)
}

Published online: 16 December 2016

(C) Springer-Verlag Berlin Heidelberg 2016

\section{Erratum to: Eur J Nucl Med Mol Imaging \\ DOI:10.1007/s00259-016-3589-9}

The author name Lida Jafari Saraf in the original version of this article should be change to Lida Jafari.

The online version of the original article can be found at http://dx.doi. org/10.1007/s00259-016-3589-9.

Silvia Morbelli

silviadaniela.morbelli@hsanmartino.it

1 Nuclear Medicine, Department of Health Science (DISSAL), University of Genoa and IRCCS AOU San Martino-IST, Largo R. Benzi 10, 16132 Genoa, Italy

2 Department of Nuclear Medicine, Clinica Universidad de Navarra, University of Navarra, Pamplona, Spain

3 Department of Nuclear Medicine, Academic Medical Center, University of Amsterdam, Amsterdam, The Netherlands

4 Radiology and Biomedical Imaging, Yale University School of Medicine, New Haven, CT, USA

5 Inserm, U1077 Caen, France

6 Université de Caen Basse-Normandie, UMR-S1077, Caen, France

7 Ecole Pratique des Hautes Etudes, UMR-S1077, Caen, France

8 CHU de Caen, U1077 Caen, France

9 Department of Radiology and Imaging Sciences, University of Utah, Salt Lake City, UT, USA
10 University of Michigan Ann Arbor, Hamden, Michigan, USA

11 QMC-Quality Medical Consulting, Hamden, Michigan, USA

12 Department of Nuclear Medicine, University of Cologne, Cologne, Germany

13 Department of Nuclear Medicine, Bagcilar Education and Research Hospital, Istanbul, Turkey

14 Department of Medical Imaging, Geneva University and Geneva University Hospitals, Geneva, Switzerland

15 Department of Nuclear Medicine, University of Leipzig, Leipzig, Germany

16 Department of Radiology, Faculty of Medicines, Kindai University, Osaka, Japan

17 Department of Radiology, Veterans Affairs Healthcare System, Greater Los Angeles, CA, USA

18 Department of Radiology \& Nuclear Medicine, VU University Medical Center, Amsterdam, The Netherlands

19 Department of Clinical Physiology, Nuclear Medicine and PET, Copenhagen University Hospital, Rigshospitalet, Copenhagen, Denmark 
$\overline{20}$ Department of Radiology, University of Texas South western Medical Center, Dallas, TX, USA

21 Departament of Nuclear Medicine, Hospital das Forças Armadas, Brasilia, Brazil

22 Institute of Cognitive Sciences and Technologies, CNR, Rome, Italy

23 Department of Nuclear Medicine, Karolinska Hospital, Stockholm, Sweden

24 Institute of Biostructure and Bioimaging, CNR, Naples, Italy

25 Department of Molecular and Medical Pharmacology, University of California, Los Angeles, CA, USA

26 Nuclear Medicine Unit, Department of Medical-Surgical Sciences and of Translational Medicine, "Sapienza", University of Rome,

Rome, Italy

27 Department of Molecular Imaging, Austin Health, Melbourne, Australia

28 Department of Medicine, University of Melbourne, Melbourne, Australia

29 Florey Institute of Neuroscience and Mental Health, University of Melbourne, Melbourne, Australia 